\title{
Phonons, electrons and thermal transport in Planckian high
} $T_{\mathrm{c}}$ materials

\author{
Connie H. Mousatov ${ }^{1}$ and Sean A. Hartnoll $\mathbb{D}^{1 凶}$
}

The room-temperature thermal diffusivity of high $T_{\mathrm{c}}$ materials is dominated by phonons. This allows the scattering of phonons by electrons to be discerned. We argue that the measured strength of this scattering suggests a converse Planckian scattering of electrons by phonons across the room-temperature phase diagram of these materials. Consistent with this conclusion, the temperature derivative of the resistivity of strongly overdoped cuprates is noted to show a kink at a little below $200 \mathrm{~K}$ that we argue should be understood as the onset of a high-temperature Planckian $T$-linear scattering of electrons by classical phonons. This kink continuously disappears toward optimal doping, even while strong scattering of phonons by electrons remains visible in the thermal diffusivity, sharpening the long-standing puzzle of the lack of a feature in the $T$-linear resistivity at optimal doping associated with the onset of phonon scattering.

npj Quantum Materials (2021)6:81 ; https://doi.org/10.1038/s41535-021-00383-w

\section{INTRODUCTION}

The thermal conductivity of conventional metals at room temperature obeys the Wiedemann-Franz law ${ }^{1}$. This establishes that heat transport is dominated by electrons and that the electronic scattering is elastic. Indeed, at these temperatures electrons are scattered elastically off classical phonons, leading to a T-linear resistivity with an underlying scattering rate of $1 / \tau \approx k_{\mathrm{B}} T / \hbar^{2}$. This rate has come to be known as "Planckian" 3 .

The thermal conductivity of unconventional metals such as high $T_{c}$ cuprates and pnictides at room temperature does not obey the Wiedemann-Franz $\operatorname{law}^{4-8}$. The Lorenz ratio is larger than the Sommerfeld value, $L>L_{0}$, often by a factor of three or more. Heat transport at room temperature in these materials is dominated by phonons rather than electrons. Nonetheless, as in conventional metals, over important parts of the phase diagram the resistivity is $T$-linear with a Planckian scattering rate ${ }^{9-14}$. As we shall see in Section 2.4 below, even away from optimal doping, the roomtemperature resistivity has a Planckian $T$-linear component. At these temperatures, phonons have every right, as in conventional metals, to be the cause of this Planckian scattering (or, at least, to contribute to it significantly).

There are good reasons to doubt the role of phonon scattering in high $T_{c}$ electrical transport, articulated forcefully in ${ }^{15}$. First, in many high $T_{\mathrm{c}}$ materials Planckian scattering continues to very low temperatures ${ }^{16}$, where phonons are presumably irrelevant (even allowing for the somewhat small Fermi surfaces of these materials, cf. ${ }^{17}$ ). Second, $T$-linear resistivity continues to very high temperatures ${ }^{18-20}$, where the short mean-free path of the electrons would seem to invalidate a naive scattering picture ${ }^{21}$. Third, the temperature and doping dependence of the resistivity across the phase diagram of high $T_{\mathrm{c}}$ materials-as well as the behavior of many other observables-reflects the importance of electronic correlations $^{22}$. A complete picture must include strong electronic correlations also, although this fact in itself does not negate a potentially important role for phonons.

In this paper, we make two observations concerning the role of electron-phonon scattering in these materials. We will be interested in intermediate temperatures, where (some) phonons are classical but where electronic mean free paths are not yet extremely small. First, in Section 2.1, we argue that at these temperatures, the scattering of phonons by electrons is visible in the large phonon contribution to heat transport in high $T_{\mathrm{c}}$ materials. This gives direct evidence for the occurrence of strong electron-phonon interactions. These same interactions are then argued to lead, conversely, to a Planckian lifetime for electrons due to scattering by phonons. Second, in Section 2.4, we note that the temperature derivative of the resistivity of heavily overdoped cuprates shows a kink at the temperature scale where scattering of electrons by classical phonons is expected to onset. We argue that this, again, demonstrates the occurrence of strong electron-phonon interactions. The kink is seen to disappear continuously toward optimal doping, offering a well-grounded angle of approach to the long-standing puzzle of the lack of features in the optimally doped resistivity due to phonon scattering.

Our emphasis on the importance of electron-phonon scattering processes for transport in high- $T_{c}$ materials at temperatures above around $200 \mathrm{~K}$ builds on the measurements and interpretation of thermal diffusivity $i^{23-25}$. Here we show how the experimental results can be understood within a quasiparticle picture of electron-phonon interactions. Furthermore, in Section 2.2, we extend the analysis of ${ }^{24}$ to different high $T_{\mathrm{c}}$ materials and dopings, using existing data for the thermal conductivity, specific heat, and electrical resistivity. In Section 2.3, we establish a parallel between high-temperature thermal and electric transport in high- $T_{c}$ materials and in heavily doped semiconductors. The role of electron-phonon scattering has been well-characterized in the semiconductors, supporting our general discussion.

\section{RESULTS}

Action and reaction of electrons and phonons

Our starting point is recent measurements of the thermal diffusivity $D_{\text {th }}$ in several cuprates near optimal doping at temperature $T$ up to $600 \mathrm{~K}^{23,24}$. Above around $200 \mathrm{~K}$, the inverse thermal diffusivity, a measure of thermal resistivity, is found to

${ }^{1}$ Department of Physics, Stanford University, Stanford, CA 94305-4060, USA. ${ }^{凶}$ email: hartnoll@stanford.edu 
behave as

$D_{\mathrm{th}}^{-1}=\frac{\lambda T}{v_{s}^{2}}+D_{0}^{-1}$,

with the temperature-independent coefficients

$\lambda \sim \lambda_{\circ} \frac{k_{\mathrm{B}}}{\hbar}, \quad D_{0}^{-1} \sim \frac{m_{\star}}{\hbar}$.

Here $v_{s}$ is the sound speed and $m_{\star}$ the effective electron mass. The dimensionless prefactor $\lambda_{\mathrm{o}} \sim 1 / 5$ in these materials ${ }^{24}$. The leading high-temperature behavior $D_{\text {th }} \sim v_{s}^{2} \times \hbar /\left(k_{\mathrm{B}} T\right)$ in (1) is characteristic of crystalline insulators with poor thermal conduction $^{25-27}$. This term is due to the degradation of the phonon heat current due to phonon anharmonicity and is entirely analogous to the $T$-linear scattering of electrons by classical phonons. The fact that the high-temperature heat transport of unconventional metals mirrors that of insulators is consistent with the dominant heat transport by phonons seen in the large Lorenz ratio, mentioned above.

The constant offset term in (1), however, is not present in crystalline insulators $^{25}$. We have already seen that the heat is carried by phonons. The inverse diffusivity is thus proportional to the phonon-scattering rate. The additive inverse diffusivity in (1) therefore immediately suggests a Matthiessen rule in which a single degree of freedom undergoes two distinct scattering mechanisms. Given that the constant term is absent in insulators, the most natural additional scattering process is that of phonons by charge carriers (more precisely, particle-hole pairs). That is, it is natural to read (1) as

$$
D_{\mathrm{th}}^{-1} \sim D_{\mathrm{th}, \mathrm{ph}}^{-1} \sim \frac{d}{v_{s}^{2}} \frac{1}{\tau_{\mathrm{ph}}}=\frac{d}{v_{s}^{2}}\left(\frac{1}{\tau_{\mathrm{ph} \rightarrow \mathrm{ph}}}+\frac{1}{\tau_{\mathrm{ph} \rightarrow \mathrm{el}}}\right),
$$

where scattering rates have been added and $d$ is the number of spatial dimensions. From the comments below (2) and with $d=3$, one has $\tau_{\mathrm{ph} \rightarrow \mathrm{ph}}^{-1} \sim \frac{1}{15} k_{\mathrm{B}} T / \hbar$. The fact that the phonons have longer lifetimes than the Planckian electrons is important for the phonons to be able to dominate thermal transport, as is the fact that the Fermi velocity is relatively small in these systems $\left(v_{\mathrm{F}} / v_{s} \approx\right.$ $\left.35^{24}\right)$. Let us now discuss $\tau_{\mathrm{ph} \rightarrow \mathrm{el}}^{-1}$.

It is a fundamental physical principle that to every action there is an equal and opposite reaction. This logic will connect the timescale $\tau_{\mathrm{el} \rightarrow \mathrm{ph}}$ for an electron to emit and reabsorb a phonon with the timescale $\tau_{\mathrm{ph} \rightarrow \mathrm{el}}$ for a phonon to decay into a particle-hole pair. These two timescales need not themselves be equal, but they are both controlled by the strength of the coupling between electrons and phonons. When a dimensionless measure of the electron-phonon coupling (the deformation potential relative to the characteristic energy scales of the electrons and phonons) is order one in magnitude, so that the electronic lifetime $\tau_{\mathrm{el} \rightarrow \mathrm{ph}}$ is Planckian, the phonon lifetime $\tau_{\mathrm{ph} \rightarrow \mathrm{el}}$ takes a characteristic value. We show in the Supplementary Material that, in agreement with well-established results for electron-phonon scattering $^{28}$,

$$
\frac{1}{\tau_{\mathrm{el} \rightarrow \mathrm{ph}}} \sim \frac{k_{\mathrm{B}} T}{\hbar} \quad \Longleftrightarrow \quad \frac{1}{\tau_{\mathrm{ph} \rightarrow \mathrm{el}}} \sim \frac{m_{\star} v_{\mathrm{s}}^{2}}{\hbar} .
$$

We have assumed that the electrons are degenerate with a large Fermi surface, so that the Fermi wavelength is comparable to the lattice spacing, $k_{\mathrm{F}} a \sim 1$, while the phonons are classical. We have neglected all numerical factors, that depend on microscopic considerations. The energy scale $m_{\star} v_{S}^{2}$ in (4) arises as the Debye energy squared over the Fermi energy: $\tau_{\mathrm{ph} \rightarrow \mathrm{el}}^{-1} \sim \hbar \omega_{\mathrm{D}}^{2} / E_{\mathrm{F}} \sim$ $m_{\star} v_{s}^{2} / \hbar$, where $E_{\mathrm{F}} \sim \hbar^{2} /\left(m_{\star} a^{2}\right)$ and $\omega_{\mathrm{D}} \sim v_{s} / a$. The result (4) also assumes that the phonon system can self-equilibrate on faster timescales than it returns momentum and energy to the charge carriers (Bloch's Annahme). At high temperatures, this is reasonable due to the strong effects of lattice anharmonicity ( $\left.\tau_{\mathrm{ph} \rightarrow \mathrm{ph}}^{-1} \gtrsim \tau_{\mathrm{ph} \rightarrow \mathrm{el}}^{-1}\right)$.

Using the second relation in (4) in the diffusivity (3) recovers precisely the observed offset in (1) and (2). Therefore, the single assumption of electron-phonon scattering with an order-one dimensionless coupling constant simultaneously leads to-at these high temperatures-both the observed Planckian charge transport and the offset to the observed Planckian heat transport. This is not a strong assumption, and holds in conventional metals.

\section{Thermal diffusivity in high- $\boldsymbol{T}_{\mathbf{c}}$ materials}

Here we extend the analysis of thermal diffusivity in ${ }^{24}$ to different families of high- $T_{c}$ materials and different dopings. We use existing thermal conductivity $k$ and specific heat $c$ data for those materials. The thermal diffusivity is then given by $D_{\text {th }}=\kappa / c$. The direct thermal diffusivity measurements in ${ }^{23,24}$ extended in several cases to $600 \mathrm{~K}$. This allowed the regime of behavior (1) to be clearly visible. We are not aware of any other published measurements of both $K$ and $c$ in metallic high- $T_{c}$ compounds up to these temperatures. Our analysis is therefore limited to temperatures up to room temperature. The onset of the behavior (1) is visible in the temperature range of $200-300 \mathrm{~K}$, but the linearity of $D_{\text {th }}^{-1}$ in $T$ is typically not fully developed. The diffusivity below $200 \mathrm{~K}$ remains dominated by phonons, but the more complicated temperature dependence means that scattering by charge carriers and by other (no longer classical) phonons is less easily separated out. In our opinion, further measurements to higher temperatures are highly desirable.

Figure 1 shows $D_{\text {th }}^{-1}$ for several materials, including underdoped LSCO and YBCO, overdoped LSCO and close-to-optimally doped $\mathrm{EBCO}$ and YBCO. We have restricted attention to compounds where electrical-resistivity measurements in the same (or very similar) crystals over the same temperature range show that the Lorenz ratio is large (see Supplementary Material). This fact suggests that phonons dominate the heat current. We will not attempt to further isolate the phonon heat current by subtracting out the electronic contribution using the Wiedemann-Franz law. Such subtractions are imprecise, even in conventional systems, and furthermore the extracted values of the intercept $D_{0}^{-1}$ are not found to change significantly. Most of the variation seen in $D_{\mathrm{th}}^{-1}=$ $c / \kappa$ comes from the specific heat, while the thermal conductivity is almost constant. This highlights the fact that $D_{\text {th }} \sim v_{s}^{2} \tau_{\text {ph }}$ is more physically transparent than $\kappa$ here: the temperature variation of $D_{\mathrm{th}}^{-1}$ directly relates to the phonon-scattering rate, showing the onset of scattering by classical phonons above $200 \mathrm{~K}$.

Figure 1 also shows the extrapolation of the high temperature linear in $T$ regime to obtain the intercept $D_{0}^{-1}$. Due to the limited temperature range of data available, the linear regime is not fully developed. In the data of ${ }^{24}$, this regime is seen to onset at around

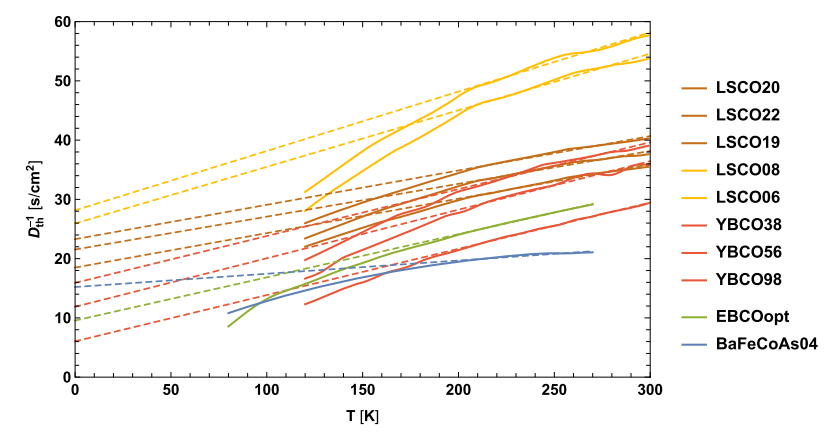

Fig. 1 Inverse thermal diffusivity for several high- $T_{c}$ compounds. Solids lines are data and the dashed lines show the intercept of the high temperature linear in $T$ regime. Within each group (overdoped and underdoped $\mathrm{LSCO}^{53,54}$, a-axis $\mathrm{YBCO}^{5,55}, \mathrm{EBCO}^{8,56}$, and $\mathrm{Ba}$ $122^{6,57,58}$ ), the curves are in the order shown in the legend. 
$200 \mathrm{~K}$. We have therefore made the linear fits in the temperature range of $200-300 \mathrm{~K}$. The intercepts are seen to lie in the range of around $10-30 \mathrm{~s} / \mathrm{cm}^{2}$, consistent with the values obtained from higher temperature diffusivity measurements $i^{24,25}$.

We now argue that the intercepts in Fig. 1 indicate a scattering rate $\tau_{\text {ph } \rightarrow \text { el }}^{-1}=\mathcal{O}(1) \times m_{\star} v_{s}^{2} / \hbar$. This is in agreement with the estimates for other materials in ${ }^{24}$ and compatible with Planckian charge carriers according to the "action-reaction" relation (4). The starting point is the fundamental quantity $m_{\mathrm{e}} / \hbar \approx 0.86 \mathrm{~s} / \mathrm{cm}^{2}$. The larger intercepts in Fig. 1 are due to two additional factors. First, the factor of $d \sim 3$ in diffusivity (3); heat diffusion is threedimensional but anisotropic. Second, the enhancement of the effective mass $m_{\star}$. In YBCO, quantum oscillations and optical measurements suggest $m_{\star} \approx 2-3 m_{\mathrm{e}}{ }^{29,30}$, while in underdoped LSCO, the effective mass is larger ${ }^{29}$, and, from specific heat measurements, significantly larger on the overdoped side: $m_{\star} \approx$ $5-10 m_{\mathrm{e}}{ }^{16}$. These mass renormalizations lead to comparable values of $D_{0}^{-1} \times \hbar / m_{\star}$ for the different compounds in Fig. 1 .

Over the temperature range where the thermal diffusivity is fit, the resistivity is metallic but is only precisely $T$-linear for the optimally doped samples ${ }^{22}$. Over this range, the product of the Fermi momentum and electronic mean-free path as extracted from the resistivity, $k_{\mathrm{F}} \ell=h / e^{2} \times d_{\mathrm{c}} / \rho$ with $d_{\mathrm{c}}$ the spacing between conducting planes, is greater than $2 \pi$ for the overdoped samples but slightly less than $2 \pi$ for the underdoped samples. A simple quasiparticle picture of charge transport may only be marginally valid in these latter cases.

The curve for the close-to-optimally electron-doped pnictide Ba122 is noticeably flatter than the others. This high-temperature behavior is suggestive of a saturated "glassy" phonon mean free path $\ell_{\mathrm{ph}}$ rather than the $\ell_{\mathrm{ph}} \sim 1 / T$ characteristic of crystalline phonons, as has been reported previously in insulating $\mathrm{BSYCO}^{4}$. Saturation may be due to strong disorder ${ }^{31}$ and/or strong lattice anharmonicity $^{32}$.

Disorder and comparison with heavily doped semiconductors The main confounding factor for our proposed interpretation of the thermal diffusivity data in terms of phonon scattering by charge carriers is the role of disorder. As we explain below, scattering of phonons by disorder also contributes to the offset $D_{0}^{-1}$. Doping introduces both disorder and charge-carrier scattering simultaneously, so, a more refined analysis is needed to tease these effects apart. Here we argue that the offset due to disorder scattering does not overwhelm that due to scattering by charge carriers.

The scattering of phonons by both disorder and charge carriers has been extensively studied in the thermal transport of heavily doped semiconductors. Heavily doped semiconductors have similarities with both high- $T_{\mathrm{c}}$ compounds and conventional materials: high-temperature thermal transport is dominated by phonons and the inverse diffusivity obeys (1), with an offset comparable to that in high- $T_{\mathrm{c}}$ materials, while electrical resistivity is $T$-linear and Planckian, due to scattering of degenerate electrons by phonons. In the Supplementary Material, we review experimental results in heavily doped $\mathrm{Ge}-\mathrm{Si}$ alloys ${ }^{33-35}$, as well as heavily doped single-silicon crystals ${ }^{36}$.

The essential point emerging from the understanding of heavily doped semiconductors is that while mass disorder effectively scatters high-frequency phonons, it does not scatter lowfrequency phonons as efficiently because the scattering needs to induce vibrations in the impurity atoms $\mathrm{s}^{37}$. The surviving longwavelength phonons that do transport heat are especially sensitive to scattering by charge carriers, because they are able to excite particle-hole pairs on the Fermi surface. This allows phonon-electron scattering to have a significant effect on phonon heat transport ${ }^{35,38,39}$.
The frequency dependence of scattering is captured by the widely employed Callaway model for thermal transport by phonons ${ }^{40,41}$, extended to include phonon-electron scattering ${ }^{35}$. This model has previously been used to estimate the role of disorder and carrier scattering in the Ge-Si alloys $\mathrm{s}^{35}$ and doped silicon $^{36}$ samples that we consider in the Supplementary Material. At high temperatures, the model gives the (three-dimensional) thermal diffusivity as

$$
D_{\text {th }, \text { ph }}=\frac{v_{s}^{2}}{3} \int_{0}^{1} \hat{\omega}^{2} \tau_{\text {ph }}(\hat{\omega}) d \hat{\omega}=\frac{v_{s}^{2}}{3} \int_{0}^{1} \frac{\hat{\omega}^{2} d \hat{\omega}}{\tau_{\text {ph,dis }}^{-1} \hat{\omega}^{4}+\tau_{\text {ph } \rightarrow \text { el }}^{-1} \hat{\omega}+\tau_{\text {ph } \rightarrow \text { ph }}^{-1} \hat{\omega}^{2}} .
$$

Here $\hat{\omega}=\omega / \omega_{D}$, with $\omega_{D}$ the Debye scale, and the denominator in the final expression contains three terms corresponding to scattering from disorder, charge carriers, and other phonons. The factor of $\hat{\omega}^{2}$ in the numerator comes from the phase space for acoustic phonons. The inverse timescales that appear in the denominator are frequency-independent. The quantities $\tau_{\mathrm{ph} \rightarrow \mathrm{el}}^{-1}$ and $\tau_{\mathrm{ph} \rightarrow \mathrm{ph}}^{-1}$ have been given previously in the text. The timescale for scattering of phonons by disordered impurities is

$\frac{1}{\tau_{\text {ph,dis }}} \sim x \omega_{\mathrm{D}}$.

Here $x$ is the density of impurities together with other factors proportional to the change of the local atomic vibrational energy due to the substitution of the atom (e.g., ${ }^{35,37}$ ).

In the asymptotic high-temperature limit, phonon scattering dominates so that $\tau_{\mathrm{ph} \rightarrow \mathrm{ph}}^{-1} \gg \tau_{\mathrm{ph}, \mathrm{dis}}^{-1}, \tau_{\mathrm{ph} \rightarrow \mathrm{el}}^{-1}$. In this limit, the integral in (5) can be performed explicitly to yield the inverse diffusivity

$$
D_{\mathrm{th}, \mathrm{ph}}^{-1}=\frac{3}{v_{\mathrm{S}}^{2}}\left(\frac{1}{\tau_{\mathrm{ph} \rightarrow \mathrm{ph}}}+\frac{1}{12} \frac{1}{\tau_{\mathrm{ph}, \mathrm{dis}}}+\frac{1}{\tau_{\mathrm{ph} \rightarrow \mathrm{el}}} \log \frac{\tau_{\mathrm{ph} \rightarrow \mathrm{el}}}{\tau_{\mathrm{ph} \rightarrow \mathrm{ph}}}+\mathcal{O}\left(\tau_{\mathrm{ph} \rightarrow \mathrm{ph}}\right)\right) .
$$

This expression amounts to a refined version of (3). Relative to an estimate that neglects frequency dependence, scattering from carriers is logarithmically enhanced, while disorder scattering is suppressed by a factor of $1 / 12$. This suppression is due to the fact that low-frequency phonons are not efficiently scattered by atomic impurities. It follows from (7) that the relative contribution of disorder and carrier scattering to the high-temperature offset $D_{0}^{-1}$ is determined, for sufficiently large Fermi surfaces, by

$\frac{x}{12} \omega_{\mathrm{D}} \quad$ vs. $\quad \frac{\hbar \omega_{\mathrm{D}}}{E_{\mathrm{F}}} \omega_{\mathrm{D}}$.

Here we neglected the logarithmic enhancement and recalled that estimate (4) came from $\tau_{\mathrm{ph} \rightarrow \text { el }}^{-1} \sim \hbar \omega_{\mathrm{D}}^{2} / E_{\mathrm{F}} \sim m_{\star} v_{s}^{2} / \hbar$. In the regime where phonons are strictly classical and charge carriers degenerate, $\hbar \omega_{\mathrm{D}} \ll k_{\mathrm{B}} T \ll E_{\mathrm{F}}$, the carrier-scattering contribution in (8) is small because $\hbar \omega_{\mathrm{D}} / E_{\mathrm{F}} \ll 1$. However, in the heavily doped semiconductors, the ratio $\hbar \omega_{\mathrm{D}} / E_{\mathrm{F}}$ is not extremely small, larger than $1 / 5$, while $x$ is order one for the alloys and at least an order of magnitude smaller for the doped silicon samples. The contribution due to scattering by electrons is not overwhelmed. Indeed, the importance of carrier scattering in these compounds is corroborated by more thorough first-principles simulation ${ }^{38,39,42}$.

While the phonon-band structure is more complicated in cuprates (c.f the discussion of complex oxides $\mathrm{in}^{25,27}$ ), comparison (8) gives a rough estimate of the relative importance of disorder and carrier scattering. The disorder caused by doping in cuprates is in between that of the silicon samples and the Ge-Si alloys, while $\hbar \omega_{\mathrm{D}} / E_{\mathrm{F}}$ is only somewhat smaller in cuprates. Thus (8) supports our claim that phonon-carrier scattering makes a significant contribution to the offset of the high-temperature inverse diffusivity in cuprates. 

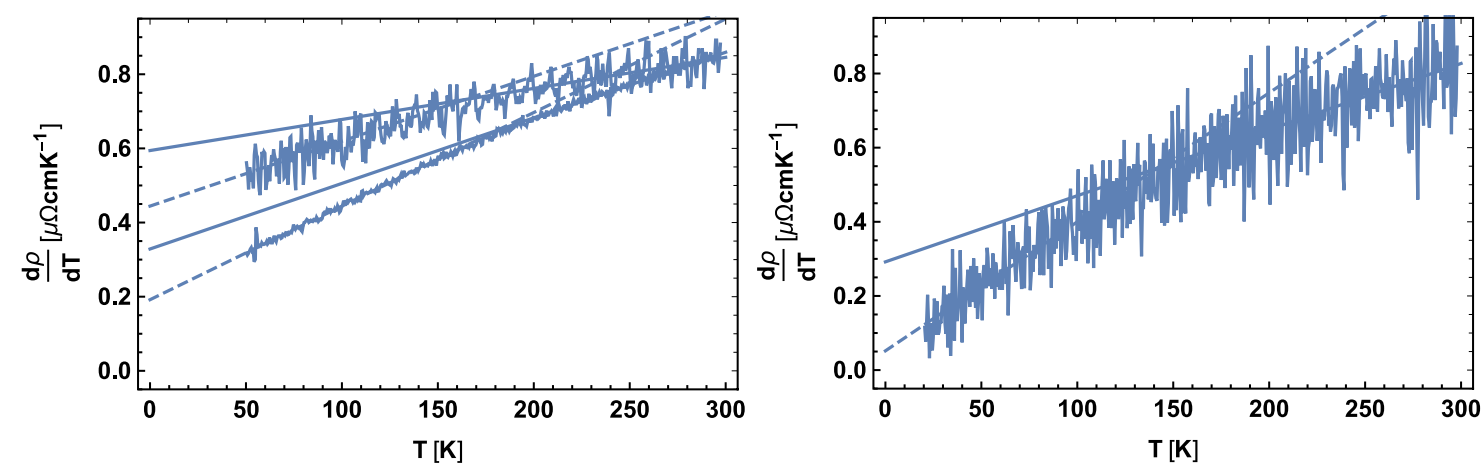

Fig. 2 Temperature derivative of the resistivity for overdoped LSCO. Left plots have $p=0.26$ (top) and $p=0.29$ (bottom). Right plot has $p=$ 0.33 . Data from ${ }^{45}$. Dashed lines are fits to $a_{\mathrm{o}}+2 b_{\mathrm{o}} T$ at low temperatures (as in ${ }^{45}$ ), while solid lines are fits to $a+2 b T$ in the temperature range of $200-300 \mathrm{~K}$

\section{Phonons and overdoped cuprates}

We have argued that the offset of the high-temperature inverse thermal diffusivity in Fig. 1 (and $\mathrm{in}^{24}$ ) is, via relation (4), suggestive of Planckian scattering of electrons by phonons across the phase diagram above about $200 \mathrm{~K}$. To see this scattering directly in electronic transport, we look to strongly overdoped samples. These are believed to have more conventional dynamics and, in particular, different scattering mechanisms can be expected to be additive. In this section, we see that the resistivity of sufficiently overdoped samples indeed shows the onset of an additive Planckian $T$-linear term at these temperatures.

Figure 2 shows that the resistivity of heavily overdoped LSCO (doping $p=0.26,0.29$, and 0.33 ) at around room temperature is of the form $\rho=\rho_{0}+a T+b T^{2}$. A kink in the temperature derivative of the resistivity is clearly discerned at a little below $200 \mathrm{~K}$. The kink implies that the room-temperature regime is not the same as the low temperature "anomalously critical" resistivity, as analyzed in, e.g., ${ }^{43,44}$. The persistence of a $T^{2}$ term indicates that this is also not the purely $T$-linear "incoherent" regime of, e.g., ${ }^{45}$. The room temperature $T+T^{2}$ behavior extends to very high temperatures at these dopings ${ }^{20}$. Given that $200 \mathrm{~K}$ is (from, e.g., our Fig. 1 and also plots of $d \rho / d T$ from Bloch-Gruneisen theory ${ }^{46}$ ) the scale at which $T$-linear phonon scattering onsets in cuprates, it is natural to understand the room-temperature regime as phonon $T$-linear plus electronic $T^{2}$ scattering. The increase in the offset in Fig. 2 above the kink indicates an additional $T$-linear scattering mechanism at high temperatures (classical phonons, we are claiming). The simultaneous decrease in the slope indicates a reduction in the $T^{2}$ scattering. This latter scattering is presumed to be electronic and the reduction may be due to the expected decrease of the electronic density of states upon crossing the phonon-energy scale. Fig. 2 shows two distinct regimes of $\rho \sim T+T^{2}$ behavior. In the low-temperature regime, the coefficient of the $T$-linear term in the resistivity (hence, the constant offset of the derivative) decreases with overdoping and becomes small at around $p=$ 0.33 , as discussed in $^{44}$ and shown in the inset of Fig. 3 . In the higher-temperature regime (fit between 200 and $300 \mathrm{~K}$ ), the $T$ linear term is instead roughly constant and saturates away from zero at large doping. This is also shown in the inset of Fig. 3. Using the estimates in ${ }^{16}$ for the effective mass and density of carriers at these dopings, the scattering timescale associated with the $T$ linear term in the high-temperature regime is found to be Planckian, with $\tau^{-1} \approx\{1,0.7,0.9\} \times k_{\mathrm{B}} T / \hbar$ for these highest three dopings, respectively. These rates are consistent with the expected Planckian scattering of electrons by phonons.

The samples in Fig. 2 are significantly more overdoped than those whose thermal transport was considered in Fig. 1. These more overdoped samples will have a larger electronic contribution to thermal transport and the analysis we performed above may not be directly applicable. The temperature derivative of the

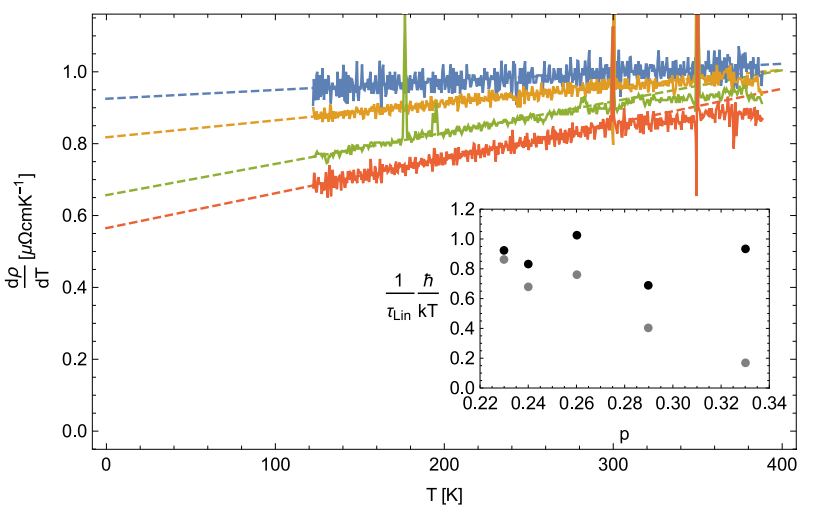

Fig. 3 Temperature derivative of the resistivity for mildly overdoped LSCO. From top to bottom $p=0.19,0.20,0.21$, and 0.22. Data from $^{22}$. Dashed lines are fits to $a+2 b T$. The inset shows the merging of the higher-temperature (upper, black dots) and lowertemperature (lower, gray dots) $T$-linear scattering rates as doping is reduced. Data for the inset from ${ }^{45}$, with the timescale expressed in Planckian units following ${ }^{16}$.

resistivity of less overdoped samples is shown in Fig. 3 , with $p=$ $0.19,0.20,0.21$, and 0.22 . Remarkably, at these lower dopings, there is no kink, and the low-temperature $\rho \sim T+T^{2}$ behavior continues up to around $300 \mathrm{~K}$ or higher. The inset of the figure shows how the kink in the $T$-linear scattering rate-which remains fixed at a little below $200 \mathrm{~K}$-disappears as doping is reduced. At the highest temperatures in Fig. 3, there is a flattening of the derivative associated with proximity to optimal doping ${ }^{20,45}$. This is distinct from the kink at lower temperature that we are discussing.

We have focussed here on overdoped LSCO, which has been the most studied in previous work. However, the same phenomenology - a feature in $d \rho / d T$ at a little below $200 \mathrm{~K}$ that disappears at small overdoping-is also seen in overdoped Bi220147.

A long-standing puzzle of optimally doped cuprates is why the $T$-linear resistivity fails to show a feature associated with the onset of scattering from classical phonons above some temperature. In Figs. 1 and 2, we have seen two instances in which such a feature is, in fact, visible. Fig. 3 furthermore shows how one of the features disappears in mildly overdoped samples, but before optimal doping is reached. This may be a fruitful angle to attack this puzzle. In particular, the presence of an additive $T^{2}$ term throughout the disappearance may shore up our confidence in a quasiparticle description. In contrast, perfectly $T$-linear resistivity at the lowest temperatures, seemingly without a $T^{2}$ term, has been reported close to a critical doping in, e.g., $\mathrm{LSCO}^{44}, \mathrm{Nd}-\mathrm{LSCO}^{48}$, $\mathrm{PCCO}^{49}$, and $\mathrm{LCCO}^{50}$. A possible explanation for the absence of ${ }^{\prime}$ feature in the resistivity close to optimal doping is a Planckian 
bound on scattering that prevents scattering channels from adding beyond a certain point ${ }^{13,14}$. If a quasiparticle description indeed holds in these mildly overdoped materials at around $200 \mathrm{~K}$, one can hope to understand in detail how nonphonon scattering below $200 \mathrm{~K}$ manages to continuously morph into phonon scattering above $200 \mathrm{~K}$.

\section{DISCUSSION}

We have argued that recent high-temperature (above $200 \mathrm{~K}$ ) measurements of thermal transport in high- $T_{c}$ materials reveal phonon scattering by charge carriers. As part of this argument, we have pointed out that the high $T_{\mathrm{c}}$ thermal diffusivity data are very similar to that in heavily doped semiconductors, where the scattering of phonons by carriers is known to be important. Interpreted in this way, the data determine the strength of electron-phonon interactions in these systems. We have shown that the interaction strength following from the data implies that the converse scattering of charge carriers by phonons leads to a Planckian lifetime for the charge carriers. This in itself is more of a consistency check than a surprise; a Planckian lifetime due to scattering by phonons at these temperatures is ubiquitous in conventional metals (and, we have noted, in heavily doped semiconductors).

However, in high- $T_{c}$ materials, electronic correlations are also important at the same high-temperature scales. It has been known for some time that charge carriers close to optimal doping have Planckian lifetimes both above and below the temperature scale at which scattering from classical phonons can occur. The slope of the resistivity does not change across that scale. From this fact, one might be tempted to draw the conclusion that electron-phonon scattering has somehow been "de-activated" by, or subsumed into, stronger electronic dynamics. Much theoretical modeling of this regime neglects phonons entirely, as do ultracold atomic simulations of the Hubbard model. From this perspective, scattering at room temperature is expected to be pure electronic close to the critical doping. On the contrary, our interpretation of the thermal diffusivity data, refining the arguments $i^{23-25}$, suggests that strong electron-phonon scattering does occur and can be seen in transport. This conclusion is consistent with the observation of strong electron-phonon interactions in angle-resolved photoemission at energy scales that are compatible with the transport phenomena we have discussed, e.g., ${ }^{51,52}$. It may be interesting to study this tension within a model that self-consistently incorporates electron-phonon scattering and the onset of electronic quantum criticality.

\section{METHODS}

As noted in the text above, equation (4) in section 2.1 follows from Fermi's Golden rule as described in the Supplementary Material. The Callaway model leading to equation (5) in section 2.3 is described explicitly in the references given immediately before that equation. All other derivations are described explicitly in the main text.

\section{DATA AVAILABILITY}

All data analyzed during the current study are available from the corresponding author on reasonable request.

Received: 27 February 2021; Accepted: 1 September 2021; Published online: 28 September 2021

\section{REFERENCES}

1. Kumar, G. S., Prasad, G. \& Pohl, R. O. Experimental determinations of the Lorenz number. J. Mater. Sci. 28, 4261-4272 (1993).

2. Peierls, R. Remarks on the theory of metals. Z. Phys. 88, 786 (1934).

3. Zaanen, J. Why the temperature is high. Nature 430, 512-513 (2004)
4. Allen, P. B., Du, X., Mihaly, L. \& Forro, L. Thermal conductivity of insulating $\mathrm{Bi}_{2} \mathrm{~S}-$ $\mathrm{r}_{2} \mathrm{YCu}_{2} \mathrm{O}_{8}$ and superconducting $\mathrm{Bi}_{2} \mathrm{Sr}_{2} \mathrm{CaCu}_{2} \mathrm{O}_{8}$ : failure of the phonon-gas picture. Phys. Rev. B 49, 9073-9079 (1994).

5. Minami, H. et al. Influence of the pseudogap on the thermal conductivity and the Lorenz number of $\mathrm{YBa}_{2} \mathrm{Cu}_{3} \mathrm{O}_{x}$ above $T_{c}$. Phys. Rev. B 68, 220503 (2003).

6. Matusiak, M. \& Wolf, T. Violation of the Wiedemann-Franz law as evidence of the pseudogap in the iron-based superconductor $\mathrm{Ba}\left(\mathrm{Fe}_{1-x} \mathrm{Co}_{x}\right)_{2} \mathrm{As}_{2}$. Phys. Rev. B 92, 020507 (2015).

7. Checkelsky, J. G. et al. Thermal Hall conductivity as a probe of gap structure in multiband superconductors: the case of $\mathrm{Ba}_{1-x} \mathrm{~K}_{x} \mathrm{Fe}_{2} \mathrm{As}_{2}$. Phys. Rev. B 86, 180502 (2012).

8. Matusiak, M. \& Wolf, T. Lorenz number in the optimally doped and underdoped superconductor $\mathrm{EuBa}_{2} \mathrm{Cu}_{3} \mathrm{O}_{y}$. Phys. Rev. B 72, 054508 (2005).

9. Collins, R. T., Schlesinger, Z., Holtzberg, F., Chaudhari, P. \& Feild, C. Reflectivity and conductivity of $\mathrm{YBa}_{2} \mathrm{Cu}_{3} \mathrm{O}_{7}$. Phys. Rev. B 39, 6571-6574 (1989).

10. Orenstein, J. et al. Frequency- and temperature-dependent conductivity in $\mathrm{YBa}_{2} \mathrm{Cu}_{3} \mathrm{O}_{6+x}$ crystals. Phys. Rev. B 42, 6342-6362 (1990).

11. Valla, T. et al. Evidence for quantum critical behavior in the optimally doped cuprate $\mathrm{Bi}_{2} \mathrm{Sr}_{2} \mathrm{CaCu}_{2} \mathrm{O}_{8+\delta}$. Science 285, 2110-2113 (1999).

12. Marel, Dvd et al. Quantum critical behaviour in a high-Tc superconductor. Nature 425, 271-274 (2003).

13. Bruin, J. A. N., Sakai, H., Perry, R. \& Mackenzie, A. P. Similarity of scattering rates in metals showing T-linear resistivity. Science 339, 804-807 (2013).

14. Hartnoll, S. A. Theory of universal incoherent metallic transport. Nat. Phys. 11, 54-61 (2015).

15. Zaanen, J. Planckian dissipation, minimal viscosity and the transport in cuprate strange metals. SciPost Phys. 6, 61 (2019).

16. Legros, A. et al. Universal T-linear resistivity and Planckian dissipation in overdoped cuprates. Nat. Phys. 15, 142-147 (2019).

17. Hwang, E. H. \& Das Sarma, S. Linear-in-T resistivity in dilute metals: a Fermi liquid perspective. Phys. Rev. B 99, 085105 (2019).

18. Gurvitch, M. \& Fiory, A. T. Resistivity of $\mathrm{La}_{1} .825 \mathrm{Sr}_{0} .175 \mathrm{CuO}_{4}$ and $\mathrm{YBa}_{2} \mathrm{Cu}_{3} \mathrm{O}_{7}$ to $1100 \mathrm{~K}$ : absence of saturation and its implications. Phys. Rev. Lett. 59, 1337-1340 (1987).

19. Martin, S., Fiory, A. T., Fleming, R. M., Schneemeyer, L. F. \& Waszczak, J. V. Normalstate transport properties of $\mathrm{Bi}_{2+x} \mathrm{Sr}_{2-y} \mathrm{CuO}_{6+\delta}$ crystals. Phys. Rev. B 41, 846-849 (1990).

20. Takagi, $\mathrm{H}$. et al. Systematic evolution of temperature-dependent resistivity in $\mathrm{La}_{2-x} \mathrm{Sr}_{x} \mathrm{CuO}_{4}$. Phys. Rev. Lett. 69, 2975-2978 (1992).

21. Emery, V. J. \& Kivelson, S. A. Superconductivity in bad metals. Phys. Rev. Lett. 74 3253-3256 (1995).

22. Ando, Y., Komiya, S., Segawa, S., Ono, S. \& Kurita, Y. Electronic phase diagram of high- $T_{c}$ cuprate superconductors from a mapping of the in-plane resistivity curvature. Phys. Rev. Lett. 93, 267001 (2004).

23. Zhang, J. et al. Anomalous thermal diffusivity in underdoped $\mathrm{YBa}_{2} \mathrm{Cu}_{3} \mathrm{O}_{6+x}$. Proc. Natl Acad. Sci. 114, 5378-5383 (2017).

24. Zhang, J. et al. Thermal diffusivity above the Mott-loffe-Regel limit. Phys. Rev. $B$ 100, 241114 (2019)

25. Zhang, J., Kountz, E. D., Behnia, K. \& Kapitulnik, A. Thermalization and possible signatures of quantum chaos in complex crystalline materials. Proc. Natl Acad. Sci. 116, 19869-19874 (2019).

26. Behnia, K. \& Kapitulnik, A. A lower bound to the thermal diffusivity of insulators. J. Phys. Cond. Mat. 31, 405702 (2019).

27. Mousatov, C. H. \& Hartnoll, S. A. On the Planckian bound for heat diffusion in insulators. Nat. Phys. 16, 579-584 (2020)

28. Ziman, J.M. The effect of free electrons on lattice conduction. Philos. Mag. 1, 191-198 (1956). Corrigendum: Philos. Mag. 2, 292 (1957).

29. Padilla, W. J. et al. Constant effective mass across the phase diagram of high- $T_{c}$ cuprates. Phys. Rev. B 72, 060511 (2005).

30. Doiron-Leyraud, N. et al. Evidence for a small hole pocket in the Fermi surface of underdoped $\mathrm{YBa}_{2} \mathrm{Cu}_{3} \mathrm{O}_{y}$. Nat. Comm. 6, 6034 (2015).

31. Cahill, D. G., Watson, S. K. \& Pohl, R. O. Lower limit to the thermal conductivity of disordered crystals. Phys. Rev. B 46, 6131-6140 (1992).

32. Sun, T. \& Allen, P. B. Lattice thermal conductivity: computations and theory of the high-temperature breakdown of the phonon-gas model. Phys. Rev. B 82, 224305 (2010)

33. Dismukes, J. P., Ekstrom, L., Steigmeier, E. F., Kudman, I. \& Beers, D. S. Thermal and electrical properties of heavily doped GeSi alloys up to 1300 K. J. Appl. Phys. 35 2899-2907 (1964).

34. Gerlich, D., Abeles, B. \& Miller, R. E. High-temperature specific heats of Ge, $\mathrm{Si}$, and Ge-Si alloys. J. Appl. Phys. 36, 76-79 (1965).

35. Steigmeier, E. F. \& Abeles, B. Scattering of phonons by electrons in germaniumsilicon alloys. Phys. Rev. 136, A1149-A1155 (1964).

36. Zhu, T. et al. The role of electron-phonon interaction in heavily doped finegrained bulk silicons as thermoelectric materials. Adv. Electron. Mater. 2, 1600171 (2016). 
37. Klemens, P. G. The scattering of low-frequency lattice waves by static imperfections. Proc. Phys. Soc. Sec. A 68, 1113-1128 (1955).

38. Liao, B. et al. Significant reduction of lattice thermal conductivity by the electronphonon interaction in silicon with high carrier concentrations: a first-principles study. Phys. Rev. Lett. 114, 115901 (2015).

39. Xu, Q., Zhou, J., Liu, T.-H. \& Chen, G. Effect of electron-phonon interaction on lattice thermal conductivity of SiGe alloys. Appl. Phys. Lett. 115, 023903 (2019).

40. Callaway, J. Model for lattice thermal conductivity at low temperatures. Phys. Rev. 113, 1046-1051 (1959).

41. Callaway, J. \& von Baeyer, H. C. Effect of point imperfections on lattice thermal conductivity. Phys. Rev. 120, 1149-1154 (1960).

42. Garg, J., Bonini, N., Kozinsky, B. \& Marzari, N. Role of disorder and anharmonicity in the thermal conductivity of silicon-germanium alloys: a first-principles study. Phys. Rev. Lett. 106, 045901 (2011).

43. Mackenzie, A. P., Julian, S. R., Sinclair, D. C. \& Lin, C. T. Normal-state magnetotransport in superconducting $\mathrm{Tl}_{2} \mathrm{Ba}_{2} \mathrm{CuO}_{6+\delta}$ to millikelvin temperatures. Phys. Rev. B 53, 5848-5855 (1996).

44. Cooper, R. A. et al. Anomalous criticality in the electrical resistivity of $\mathrm{La}_{2}-$ $x \mathrm{Sr}_{x} \mathrm{CuO}_{4}$. Science 323, 603-607 (2009).

45. Hussey, N. E. et al. Dichotomy in the T-linear resistivity in hole-doped cuprates. Philos. Trans. R Soc. A 369, 1626-1639 (2011).

46. Pickett, W. E. Temperature-dependent resistivity from phonons in cuprate superconductors. J. Supercond. 4, 397-407 (1991).

47. Putzke, C. et al. Reduced hall carrier density in the overdoped strange metal regime of cuprate superconductors. Nat. Phys. 17, 826-831 (2021).

48. Daou, R. et al. Linear temperature dependence of resistivity and change in the fermi surface at the pseudogap critical point of a high-tc superconductor. Nat. Phys. 5, 31-34 (2009).

49. Fournier, P. et al. Insulator-metal crossover near optimal doping in $\mathrm{Pr}_{2-x} \mathrm{Ce}_{x} \mathrm{CuO}_{4}$ : anomalous normal-state low temperature resistivity. Phys. Rev. Lett. 81, 4720-4723 (1998).

50. Jin, K., Butch, N. P., Kirshenbaum, K., Paglione, J. \& Greene, R. L. Link between spin fluctuations and electron pairing in copper oxide superconductors. Nature 476, 73-75 (2011).

51. Zhou, X. J. et al. Universal nodal fermi velocity. Nature 423, 398-398 (2003).

52. He, Y. et al. Rapid change of superconductivity and electron-phonon coupling through critical doping in Bi-2212. Science 362, 62-65 (2018).

53. Yan, J.-Q., Zhou, J.-S. \& Goodenough, J. B. Thermal conductivity of $\mathrm{La}_{2}-x \mathrm{Sr}_{x} \mathrm{CuO}_{4}$ (0.05 $\leq x \leq 0.22)$. New J. Phys. 6, 143-143 (2004).

54. Loram, J., Mirza, K., Cooper, J., Athanassopoulou, N. \& Liang, W., Thermodynamic evidence on the superconducting and normal state energy gaps in $\mathrm{La}_{2}-x \mathrm{Sr}_{x-}$ $\mathrm{CuO}_{4}$, in Proceedings of 10th Anniversary HTS workshop, World Scientific, Singapore, p. 341 (1996).

55. Loram, J., Mirza, K. \& Freeman, P. The electronic specific heat of $\mathrm{YBa}_{2}$ $\left(\mathrm{Cu}_{1-x} \mathrm{Zn}_{x}\right)_{3} \mathrm{O}_{7}$ from $1.6 \mathrm{~K}$ to $300 \mathrm{~K}$. Phys. C Super. 171, 243-256 (1990).

56. Bednarz, G., Stroink, G. \& White, M. A. A study of the heat capacity of the superconductor $\mathrm{EuBa}_{2} \mathrm{Cu}_{3} \mathrm{O}_{7}-x$. Phys. C Super. 165, 385-390 (1990).

57. Bud'ko, S. L., Ni, N., Nandi, S., Schmiedeshoff, G. M. \& Canfield, P. C. Thermal expansion and anisotropic pressure derivatives of $T_{c}$ in $\mathrm{Ba}\left(\mathrm{Fe}_{1-x} \mathrm{Co}_{x}\right)_{2} \mathrm{As}_{2}$ single crystals. Phys. Rev. B 79, 054525 (2009).
58. Storey, J. G., Loram, J. W., Cooper, J. R., Bukowski, Z. \& Karpinski, J. Electronic specific heat of $\mathrm{Ba}_{1-x} \mathrm{~K}_{x} \mathrm{Fe}_{2} \mathrm{As}_{2}$ from 2 to 380 K. Phys. Rev. B 88, 144502 (2013).

\section{ACKNOWLEDGEMENTS}

We are grateful to J.R. Cooper, N. Hussey, and S. Komiya for sending us raw data. We have benefited from discussions with Kamran Behnia, Steve Kivelson, Andy Mackenzie, Akshat Pandey, and Veronika Sunko. S.A.H. is supported by a Simons Investigator award. C.H.M. is supported by an NSF graduate fellowship. S.A.H. acknowledges the hospitality of the Max Planck Institute CPfS.

\section{AUTHOR CONTRIBUTIONS}

Both authors contributed to the ideas, calculations, and writing of the paper.

\section{COMPETING INTERESTS}

The authors declare no competing interests.

\section{ADDITIONAL INFORMATION}

Supplementary information The online version contains supplementary material available at https://doi.org/10.1038/s41535-021-00383-w.

Correspondence and requests for materials should be addressed to Sean A. Hartnoll.

Reprints and permission information is available at http://www.nature.com/ reprints

Publisher's note Springer Nature remains neutral with regard to jurisdictional claims in published maps and institutional affiliations.

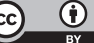

Open Access This article is licensed under a Creative Commons Attribution 4.0 International License, which permits use, sharing, adaptation, distribution and reproduction in any medium or format, as long as you give appropriate credit to the original author(s) and the source, provide a link to the Creative Commons license, and indicate if changes were made. The images or other third party material in this article are included in the article's Creative Commons license, unless indicated otherwise in a credit line to the material. If material is not included in the article's Creative Commons license and your intended use is not permitted by statutory regulation or exceeds the permitted use, you will need to obtain permission directly from the copyright holder. To view a copy of this license, visit http://creativecommons. org/licenses/by/4.0/.

(c) The Author(s) 2021 